\title{
Optimization of a Pin Surface as a Solution to Suppress Cavity Modes in a Packaged W-Band Microstrip Receiver
}

\author{
Ainara Rebollo, Ramón Gonzalo, Member, IEEE, and Iñigo Ederra
}

\begin{abstract}
A pin surface structure has been used in the upper metal plate of a packaged W-band RF front-end receiver in order to suppress the propagation of unwanted modes. A full parametric analysis of the periodic structure within the irreducible Brillouin zone has been carried out in order to understand the behavior of the pin surface and determine the optimum dimensions for its operation in the full W-band. Resonances suppression has been demonstrated in the $\mathrm{W}$-band both in simulation and experimentally.
\end{abstract}

Index Terms - Lid of nails, microstrip packaging, pin surface, $\mathrm{W}$-band receiver.

\section{INTRODUCTION}

$\mathbf{P}$ ACKAGING of millimeter-wave microstrip circuits can be seriously affected by excitation of cavity modes and surface modes in the dielectric substrate. These may penalize the transmission of the quasi-TEM mode in the microstrip circuit [1] and degrade the circuit performance.

Traditionally, conventional microwave absorbers composed of materials with bulk resistive properties have been placed within the cavity of the packaged circuits in order to avoid the propagation of cavity modes [2]. However, the circuit performance may degrade because the undesired modes are not prevented but absorbed.

The aforementioned approach causes undesired losses, which become more relevant as frequency increases. A more elegant solution to this problem can be found in the use of periodic structures in one of the ground plates of the packaging structure [3]-[8]. The used periodic textured surfaces implement an artificial magnetic conductor (AMC) or a high-impedance boundary, which blocks parallel-plate mode propagation within a frequency band (stopband) provided that the distance to the opposite perfect electric conductor (PEC) plate is smaller than a quarter wavelength.

Besides the AMC behavior, these 2-D periodic structures act as an electromagnetic bandgap (EBG), which blocks

Manuscript received September 17, 2013; revised December 26, 2013; accepted March 10, 2014. This work was supported by the Spanish Ministry of Science and Innovation under Project TEC2009-11995, Project IPT-20110960-390000, and Project CONSOLIDER CSD2008-00066. Recommended for publication by Associate Editor L.-T. Hwang upon evaluation of reviewers' comments.

The authors are with the Department of Electrical and Electronic Engineering, Public University of Navarra, Pamplona 31006, Spain (e-mail: ainara.rebollo@unavarra.es; ramon@unavarra.es; inigo.ederra@unavarra.es).

Color versions of one or more of the figures in this paper are available online at http://ieeexplore.ieee.org.

Digital Object Identifier 10.1109/TCPMT.2014.2312252 the propagation of electromagnetic waves for all directions within a forbidden frequency band [4]. Different kinds of EBG structures have been proposed for packaging microstrip circuits at microwave frequencies. Periodic structures, such as mushroom-type EBG [5], Fakir's bed of nails [6], and bed of springs [7], are more suitable for relatively low frequencies, below $10 \mathrm{GHz}$, due to its easy manufacturing and low weight. As a matter of fact, when frequency increases, the bed of spring becomes too complex to be manufactured. Moreover, the drawback of the first two cases is to use a dielectric substrate since it adds losses and reduces the operating frequency bandwidth.

The difficulty of scaling to higher frequencies these periodic structures has led to other configurations being proposed. On the one hand, in [8], an EBG structure composed of holes with period half the guided wavelength has been implemented in the upper metal plate of a shielded microstrip structure. This configuration allows the suppression of undesired cavity modes in a narrow stopband centered at $76 \mathrm{GHz}$. On the other hand, the new gap waveguide presented in [9] and experimentally validated around $15 \mathrm{GHz}$ in [10] implements a periodic structure, which consists of a lid of nails or a pin surface. Its main advantages are the omission of the dielectric substrate and its large bandwidth, up to an octave, demonstrated in [11]. Furthermore, this structure is easily scalable to higher frequencies. As shown in [12], manufacturing of this structure is feasible up to $340 \mathrm{GHz}$ with microelectromechanical system technology.

In this paper, the optimization of the pin surface for packaging of a microstrip circuit covering the full W-band is presented. By means of a parametric study, a deep understanding of the behavior of its modes and the influence of the different parameters on the performance, mainly on the bandwidth, is for the first time achieved. Moreover, in contrast to previous studies, our design takes into account for the first time any direction of the propagating modes. This allows to determine accurately the stopband of this periodic structure. So far, the pin structure has been used in packaging of microstrip circuits, such as the feeding network of the eight ports of the 11 antenna in a broad frequency range from 5 to 13.5 GHz [13]. Besides, when used together with microstrip circuits, a significant improvement of their performance can be achieved. For example, critical microstrip passive devices, such as filters, have benefit from this technology [14]. In addition, the improvement of isolation between two 


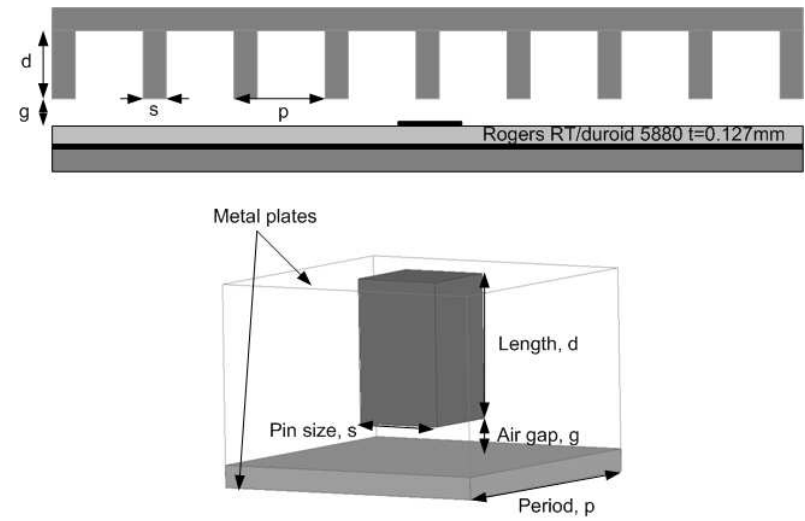

Fig. 1. Lateral view of a pin surface structure and the unit cell of a square lattice configuration of pins and its parameters.

high-gain amplifier chains in Ku-band implementing this packaging alternative has been demonstrated in [15]. Moreover, the removal of resonances for the case of a single microstrip line with two $90^{\circ}$ bends around $15 \mathrm{GHz}$ using the bed of nails has been shown in [16].

This paper optimizes the performance of a square pin surface covering the full W-band. This surface will be used for packaging of a microstrip circuit on $127-\mu \mathrm{m}$ thick Rogers RT/Duroid 5880 substrate [17]. The use of the pin surface will allow suppression of the unwanted modes and resonances. A stopband covering the full W-band has been designed by means of a complete parametric study. In this analysis, the irreducible Brillouin zone of the dispersion diagram has been taken into account. Therefore, all propagating modes in any direction within the structure are completely studied. By contrast, in most of the papers, only the transverse direction is studied. This causes that the limits of the stopband are not determined accurately. Finally, the removal of the undesired modes is demonstrated both in simulation with Ansoft HFSS and experimentally by measurements of a prototype.

\section{Pin Surface: Geometry and Principle OF OPERATION}

The geometry of a pin surface is shown in Fig. 1. The pin surface implemented in the upper metal of the package covers the microstrip circuit keeping an air gap between the end of the pin and the substrate. The pins are arranged in a square lattice configuration. The parameters that determine its performance are as follows:

1) the length of the pins, $d$;

2) the period of the structure, $p$;

3) the size of the square pin, $s$;

4) the height of the air gap between the AMC surface and the opposite substrate, $g$.

In principle, if the period is small enough, the pins work as an AMC when its length, $d$, is approximately $\lambda / 4$. Due to this length, the short circuit (PEC) is transformed into an open circuit (AMC).

When an AMC is introduced in the upper metal of two parallel metal plates separated by an air gap, it creates an ideal parallel plate cutoff, i.e., a stopband. The behavior of

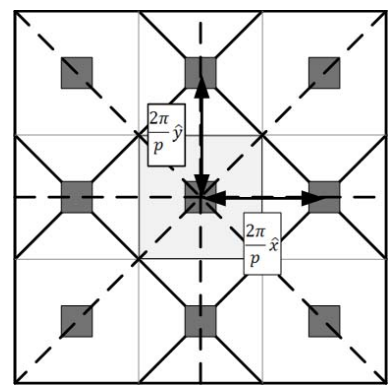

(a)

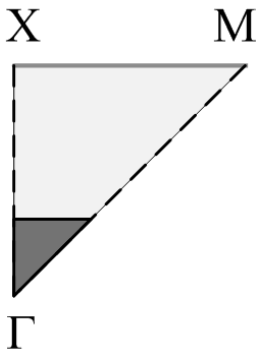

(b)
Fig. 2. (a) Schematic diagram of the Brillouin zone in a square lattice configuration of pins (gray squares) and (b) irreducible Brillouin zone.

this structure has one physical limit for the lower frequency and two physical limits for the upper frequency of the stopband [11]. On the one hand, the lower limit is defined by the frequency at which the surface impedance changes from inductive to capacitive. At this point, the surface presents a high enough impedance to generate the cutoff of the propagation modes. On the other hand, the upper limit of the stopband comes from both the impedance change from capacitive to inductive and, besides, the height of the air gap. Moreover, the distance between the plates must be smaller than half the wavelength in order to create the parallel plate cutoff.

Therefore, when a pin surface is introduced, the stopband is theoretically generated from the frequency at which the length of the pins is approximately $\lambda / 4$ up to the frequency at which the spacing between parallel metal plates is half the wavelength [10]. However, these limits are more flexible due to the capacitive effect of the air-gap height.

Besides the AMC effect, periodicity of the pin surface plays an important role in its behavior. As in any periodic structure, it will have a frequency range where no propagation is allowed. This will be the range of interest for our packaging application.

\section{Parametric Study of the Pin Surface}

For the complete definition of the stopband, it is required to determine the propagating modes in any direction within the periodic structure. This study reduces to the edge of the irreductible Brillouin zone, where information about propagation in any direction in the $k$-space is taken into account [18]. For a square lattice, the irreductible Brillouin zone is the triangular wedge in the upper right corner within the unit cell, as can be observed in Fig. 2. The rest of the Brillouin zone [central unit cell in Fig. 2(a)] consists of exact replicas of this wedge. The three points that determine the irreductible Brillouin zone, $\Gamma, X$, and $M$, correspond to $k_{\|}=0, k_{\|}=\pi / p \hat{x}$, and $k_{\|}=\pi / p \hat{x}+\pi / p \hat{y}$, respectively.

In order to understand the behavior of the pin structure and determinate the limits of the stopband, the dispersion diagrams of the pin surface have been calculated using Ansoft HFSS.

As mentioned above, the first step to study the cutoff properties of a lid of pins working as an AMC surface is to fix the pin length $d$ equal to $\lambda_{c} / 4$. In this case, $\lambda_{c}$ is the wavelength of the central frequency of the W-band. This frequency is $92.5 \mathrm{GHz}$, and therefore the fixed length of the pins is $0.81 \mathrm{~mm}$. The other parameters of the initial periodic 


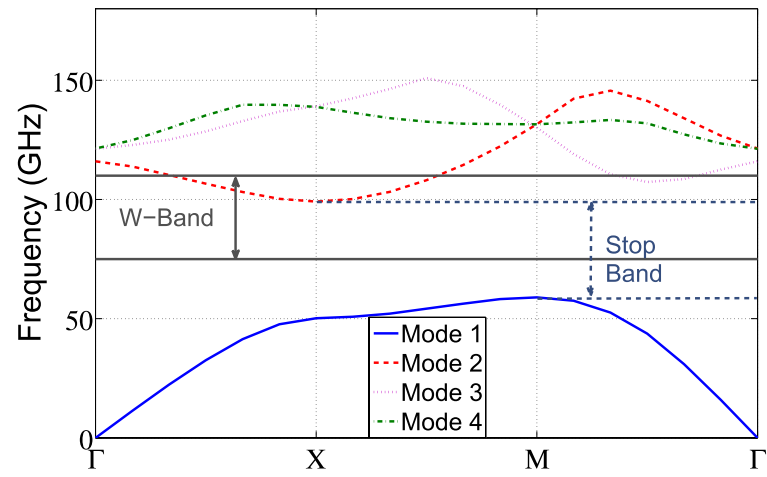

Fig. 3. Dispersion diagram of the pin surface covering a microstrip circuit over $127-\mu \mathrm{m}$ thick Rogers RT/Duroid 5880 substrate. The length and the size of the pin are 0.81 and $0.5 \mathrm{~mm}$, respectively. The period and the height of the air gap are 1.6 and $0.2 \mathrm{~mm}$, respectively.

structure have been fixed to $p=1.6 \mathrm{~mm}\left(\lambda_{c} / 2\right), s=0.5 \mathrm{~mm}$, and $g=0.2 \mathrm{~mm}$.

The dispersion diagram of the first four propagation modes for this pin surface structure can be observed in Fig. 3. According to this figure, the stopband of the periodic structure is determined by the gap between the two lowest order modes of the dispersion diagram. The highest frequency of the firstorder mode defines the lower limit. Note that this highest frequency corresponds to the $M$ point. This shows that the analysis covering the full Brillouin zone is required in order to determine the structure performance. On the other hand, the upper limit is defined by the lowest frequency of the second mode, which is in propagation within the W-band.

The normalized E-field magnitude of the first three propagating modes defined at the $X$ point is shown in Fig. 4. This figure helps to understand the nature of each propagating mode inside the structure. As can be seen, the E-field of the first propagating mode is mainly localized in the air gap under the pin, but its field distribution in the vertical direction depends on the total height. Conversely, the second mode mainly propagates in the spacing between pins.

This paper will highlight some peculiarities of the modal behavior that are of great interest for the structure design. In particular, the effects of the period, the pin size, and the height of the air gap will be linked to the mode structure. This analysis will allow to optimize the stopband of the periodic structure to cover the full W-band. For this paper, only the points of the band diagram that define the bandgap will be plotted.

\section{A. Effect of the Period}

Since the bandgap of the initial pin surface is located at frequencies lower than the $\mathrm{W}$-band, reducing its period would shift it toward higher frequencies. The dependence of the bandgap with the period is shown in Fig. 5, where the gap limits are given by the dashed and solid black lines plotted in this figure. The lower limit of the gap behaves as expected in any periodic structure, i.e., its frequency decreases when the period increases. To obtain a lower frequency limit of the stopband smaller than the lowest frequency of W-Band, i.e., $75 \mathrm{GHz}$, the period should be higher than $1 \mathrm{~mm}$,

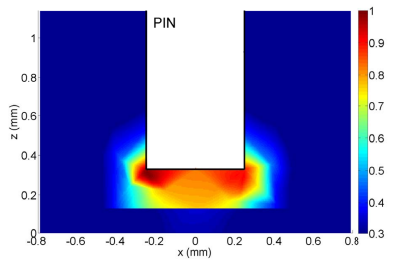

(a)

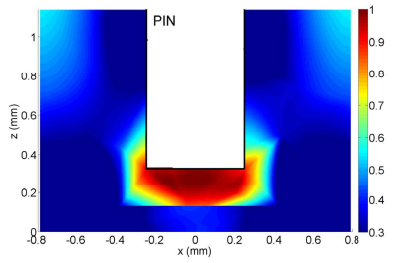

(c)

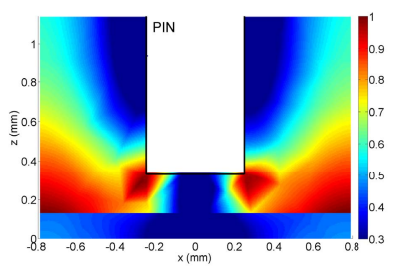

(e)

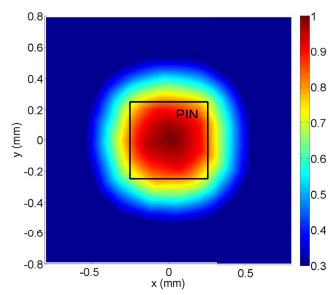

(b)

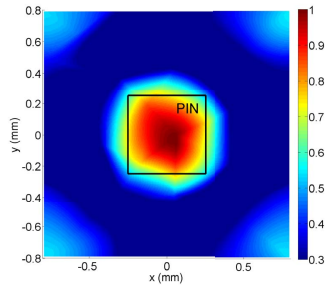

(d)

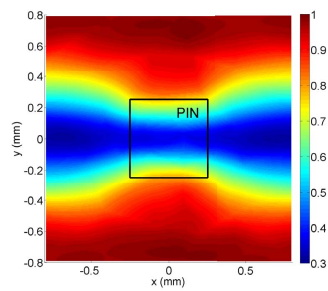

(f)
Fig. 4. Normalized E-field magnitude of the first two propagating modes defined in point of interest. (a) First mode at the center of the unit cell at point $M$. (b) First mode on the substrate surface at point $M$. (c) Second mode at the center of the unit cell at point $\Gamma$. (d) Second mode on the substrate surface at point $\Gamma$. (e) Second mode at the center of the unit cell at point $X$. (f) Second mode on the substrate surface at point $X$. The black rectangle shows the dimensions of the pin.

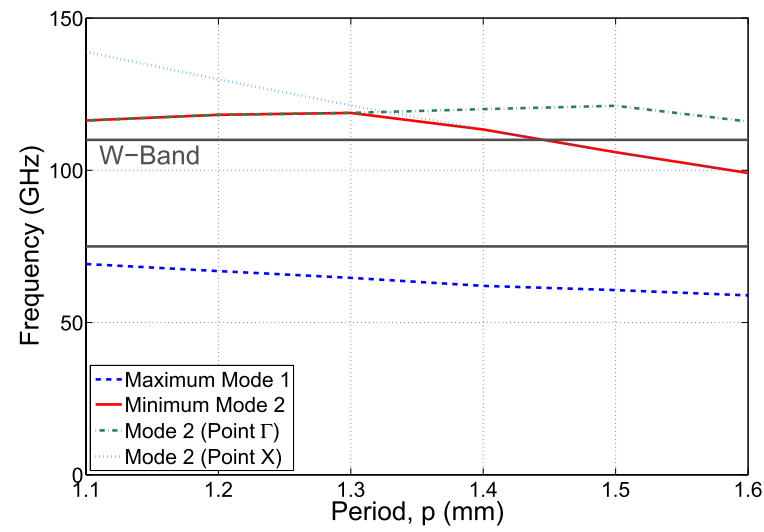

Fig. 5. Highest (second propagation mode) and lowest (first propagation mode) limits of the bandgap depending on the period. The length and the size of the pin, and the height of the air gap are 0.81 and 0.5 , and $0.2 \mathrm{~mm}$, respectively.

which corresponds to a quarter wavelength at this frequency. However, the upper limit of the gap behaves differently.

As can be seen, depending on the period, the lowest frequency of the second-order mode is determined by the point $X$ or $\Gamma$. The behavior of this higher limit of the bandgap has a maximum when the period is $1.3 \mathrm{~mm}$, which corresponds to a distance between pins of $\lambda_{c} / 4$. When the period is larger than this value, the upper frequency limit of the stopband decreases 


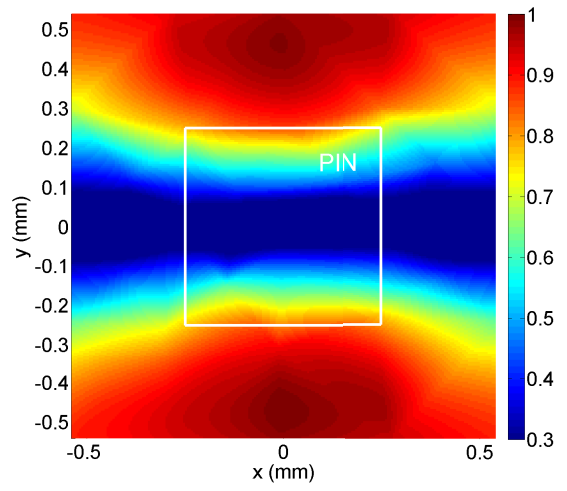

(a)

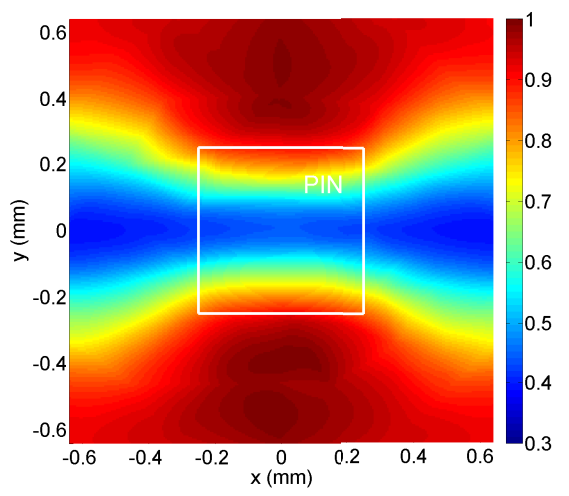

(b)

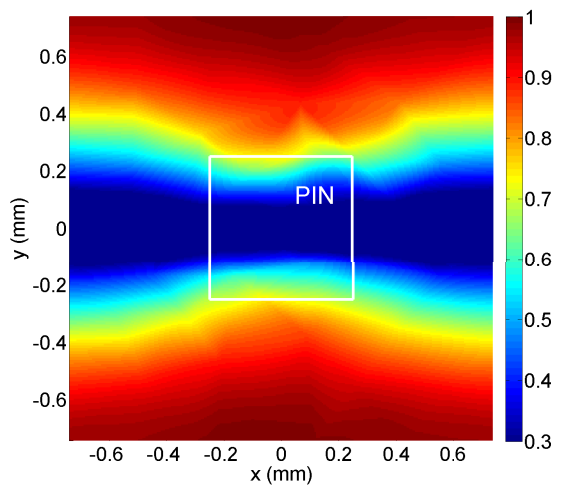

(c)

Fig. 6. Normalized E-field magnitude of the second order at the $X$ point on the substrate surface for different values of the period (a) 1.1, (b) 1.3, and (c) $1.5 \mathrm{~mm}$. The white rectangle shows the dimensions of the pin.

following the behavior of the mode for the $X$ point direction due to the increase of the spacing between pins. Fig. 6 shows the normalized E-field magnitude of the second mode at the $X$ point for different values of the period. As can be seen, the second mode propagates within the air region between pins. Therefore, when the period increases while keeping constant the size of the pin, the spacing between pins increases and the frequency at which this mode is propagated decreases.

On the other hand, below this maximum, the mode at $\Gamma$ point direction defines the upper limit of the stopband. At $\Gamma$, the mode frequency remains nearly constant. This can be explained by the field distribution of the mode (Fig. 7). In this figure, it can be seen that the maximum of the

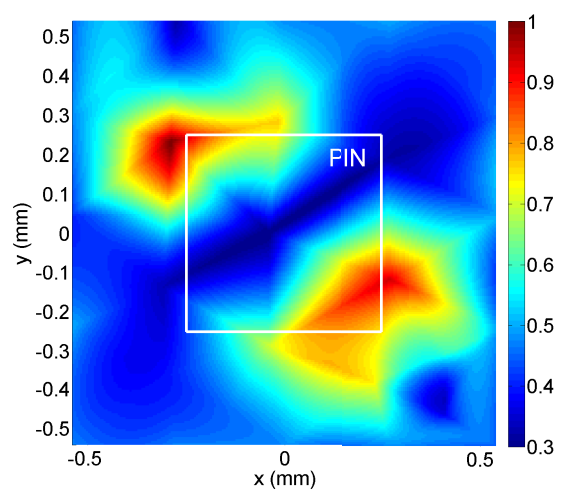

(a)

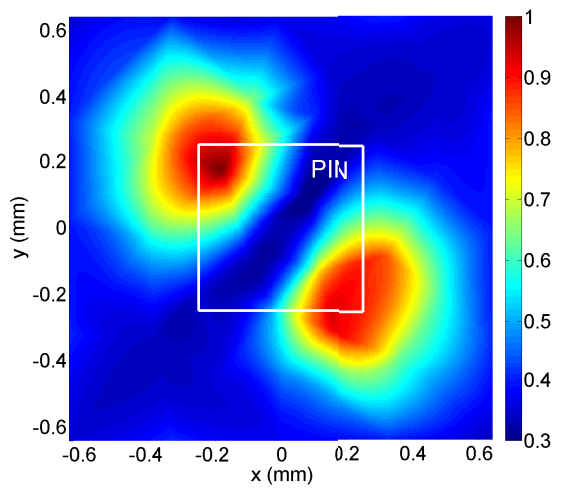

(b)

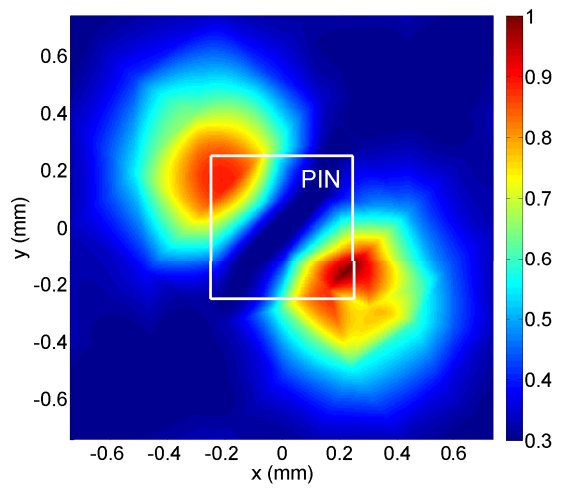

(c)

Fig. 7. Normalized E-field magnitude of the second mode at the $\Gamma$ point on the substrate surface for different values of the period (a) 1.1, (b) 1.3, and (c) $1.5 \mathrm{~mm}$. The white rectangle shows the dimensions of the pin.

E-field at point $\Gamma$ is located on the edges of the pin for three different periods. As a consequence, its frequency is mainly determined by the pin size and it is not affected by the period. In this case, the optimum period in terms of largest bandwidth corresponds to the point, where the frequency of the second mode at $X$ equals the frequency of the mode at $\Gamma$. This will be the limit for the highest frequency of the gap. This optimum period corresponds to $1.3 \mathrm{~mm}$.

\section{B. Effect of the Pin Size}

The study of the pin size has been carried out while keeping constant the period of the pin surface as $1.3 \mathrm{~mm}$. Similarly, 


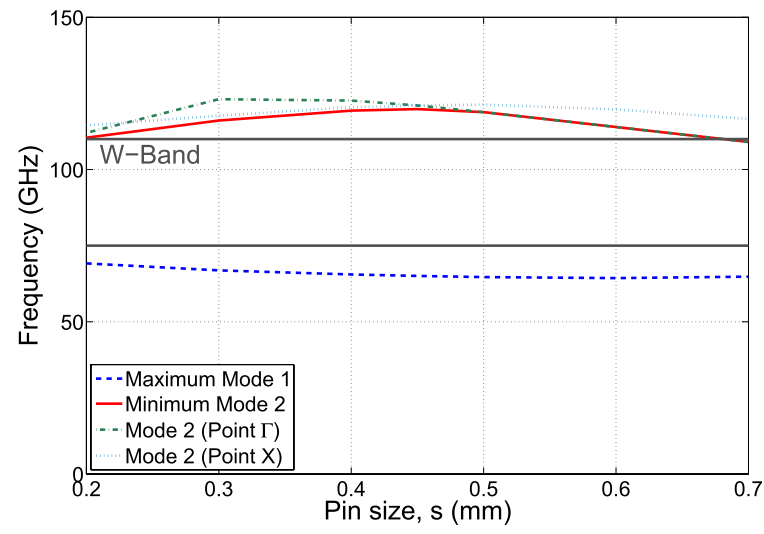

Fig. 8. Highest (second propagation mode) and lowest (first propagation mode) limits of the bandgap depending on the pin size. The length of the pin, and the period and the height of the air gap are 0.81 and 1.3 , and $0.2 \mathrm{~mm}$, respectively.

the pin size mainly affects the upper limit of the stopband, as shown in Fig. 8. As previously mentioned, the upper limit can be given by the $\Gamma$ or $X$ point, the value at $\Gamma$ being always the maximum achievable value. The largest bandwidth is achieved when the size of the pin is $0.45 \mathrm{~mm}$, which corresponds approximately to one-third of the period. Below this value, the gap decreases because of the increase of the spacing between pins. The $\Gamma$ point defines the upper limit of the stopband when the pin size is larger than $0.45 \mathrm{~mm}$, as happened with the period. In this case, increasing the size of the pin over this value means decreasing the pin spacing below $\lambda_{c} / 4$. Nevertheless, the upper limit of the bandgap decreases more steeply than when period changes, since the pin size begins to be wider than the distance between pins. Therefore, coupling effects are stronger. To summarize the effect of the pin size on the pin surface performance, the propagation frequency of the second-order mode at $X$ point equals the maximum achievable frequency when the pin size is approximately $0.34 \times$ the pin period, as shown in Fig. 8.

\section{Effect of the Air Gap}

Once the period and the pin size have been fixed to 1.3 and $0.45 \mathrm{~mm}$, respectively, the next step is to analyze the effect of the air-gap height. According to Fig. 9, the larger the air gap, the narrower the stopband due to the lower capacitive effects between the AMC surface and the metal plate. Consequently, a $0.2-\mathrm{mm}$ air gap is enough to ensure the desired operation bandwidth of the periodic structure, covering the full W-band. Moreover, this value is large enough to ensure that the pin surface does not affect the transmission of the quasi-TEM mode of the microstrip circuit.

\section{Effect of the Pin Length}

The analysis of period, pin size, and air-gap height have been carried out assuming that the length of the pins should be $\lambda_{c} / 4$ to work as an AMC. However, the analysis of the pin length has been considered in order to explain its contribution in the determination of the stopband of the structure.

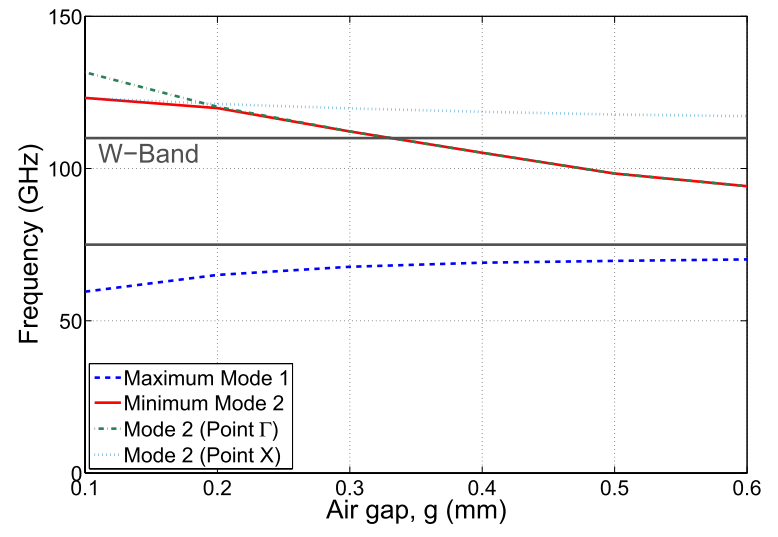

Fig. 9. Highest (second propagation mode) and lowest (first propagation mode) limits of the bandgap depending on the size of the air gap height. The length and the size of the pin, and the period are 0.81 and 0.45 , and $1.3 \mathrm{~mm}$, respectively.

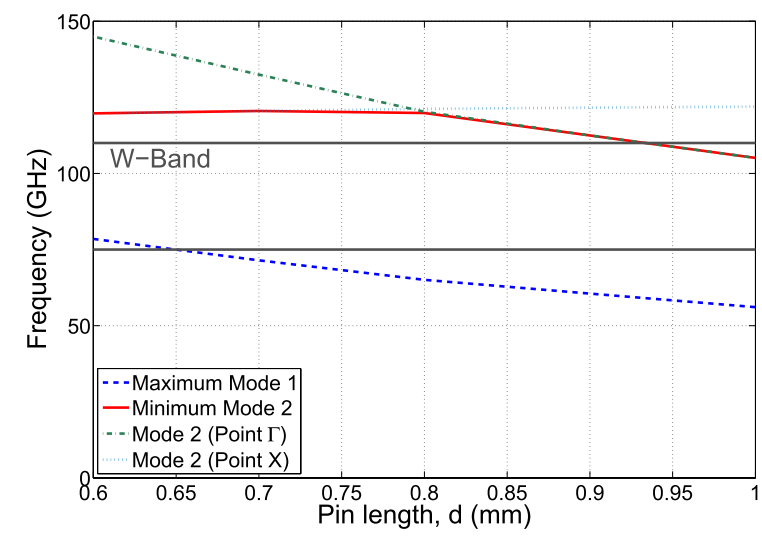

Fig. 10. Highest (second propagation mode) and lowest (first propagation mode) limits of the bandgap depending on the length of the pin. The period, the pin size, and the air-gap height are $1.3,0.45$, and $0.2 \mathrm{~mm}$, respectively.

The variation in the length of the pin mainly alters the lowest frequency limit of the stopband (Fig. 10). The shorter the pin, the higher the lowest limit. This agrees with the fact that mode 1 is determined by the unit cell total dimensions. Moreover, the dependence of the highest frequency limit of the stopband with respect to the distance between the parallel metal plates and the period can also be observed in Fig. 10. When the length of the pin increases over $0.81 \mathrm{~mm}$, the highest frequency limit decreases since it is determined by mode 2 at $\Gamma$, which depends on the height of the cavity. Note that the structure has been optimized based on this length and this result is consistent with it. However, below this value, the highest limit of the stopband is constant because it is limited by mode 2 at $X$, which depends on the period. As mentioned before, the highest achievable frequency of the stopband is defined by mode 2 at $\Gamma$ point. Therefore, as shown by the propagation frequency of the first mode and of the second mode at this point, plotted in Fig. 10, the pin length is the pin surface parameter that defines the center frequency of the stopband.

The dispersion diagram of the first four propagation modes for the optimum pin surface structure whose dimensions are $d=0.81 \mathrm{~mm}, p=1.3 \mathrm{~mm}, s=0.45 \mathrm{~mm}$, and $g=0.2 \mathrm{~mm}$ 


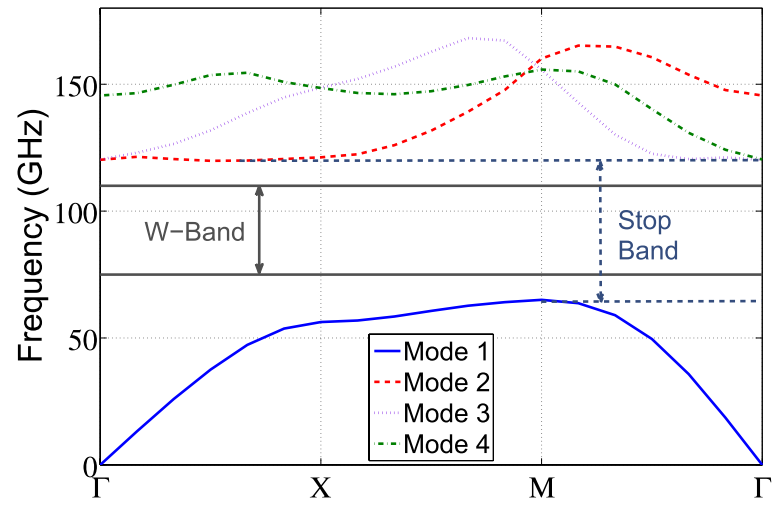

Fig. 11. Dispersion diagram of the optimum pin surface covering a microstrip circuit over $127-\mu \mathrm{m}$ thick Rogers RT/Duroid 5880 substrate. The length and the size of the pin, and the height of the air are 1.3 and 0.45 , and $0.2 \mathrm{~mm}$, respectively.

and its stopband covering the full W-band have been plotted in Fig. 11. As it can be observed, the stopband is larger than the W-band complying with the design goals.

\section{E. Design Guidelines}

Taking into account the results above, some design guidelines can be proposed for the pin structure in order to create a certain stopband.

1) Fix the length of the pins at $\lambda_{c} / 4$, where $\lambda_{c} / 4$ corresponds to the central frequency of the stopband.

2) The total height, i.e., $d-t \sqrt{\epsilon_{r}}+g$ must be smaller than $\lambda_{u} / 2$, where $\lambda_{u}$ is the wavelength at the highest frequency of the stopband. As a rule of thumb, the minimum value of the gap height is the thickness of the substrate to prevent affecting the transmission of the quasi-TEM mode of the microstrip line.

3) The period of the pin surface must be chosen inside the range $\lambda_{u} / 2<p<\lambda_{l} / 4$, where $\lambda_{l}$ is the wavelength of the required lower frequency limit of the stopband. The limits of this range make the maximum feasible frequency limit of the stopband smaller than $2 \times$ the lower frequency limit.

4) Once the period is fixed, the pin size can be selected in the range from 0.3 to $0.38 \times$ the pin period in order to obtain the maximum achievable propagation frequency of the second mode at $X$ point.

\section{Structure Performance}

The optimization of the pin surface has been realized in order to remove the resonances produced when packaging of a W-band microstrip receiver proposed in [19]. The receiver uses a Rogers RT/Duroid 5880 substrate $\left[\epsilon_{r}=2.2 ; \tan \delta=0.0009\right.$ $(10 \mathrm{GHz})]$ and is based on a differential configuration. By a combination of two microstrip quadrature hybrids couplers, both reference signal and unknown RF signal are distributed along two amplifier chains and are finally separated in order to compensate the gain and noise temperature fluctuations, as described in [17].

The demonstration that this periodic structure is able to suppress the propagation of unwanted modes has been done

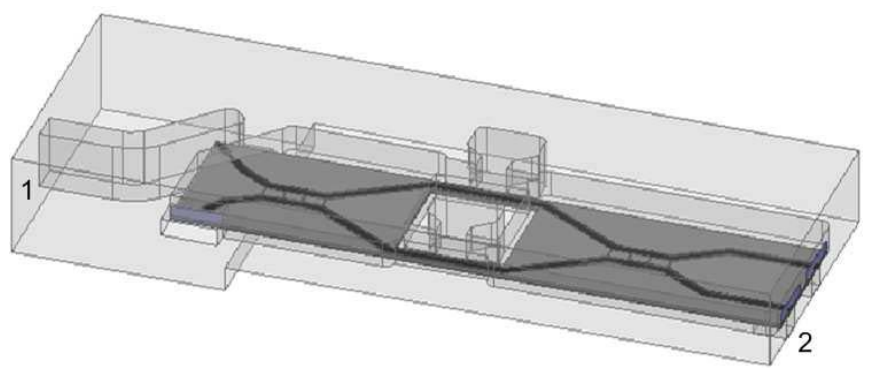

(a)

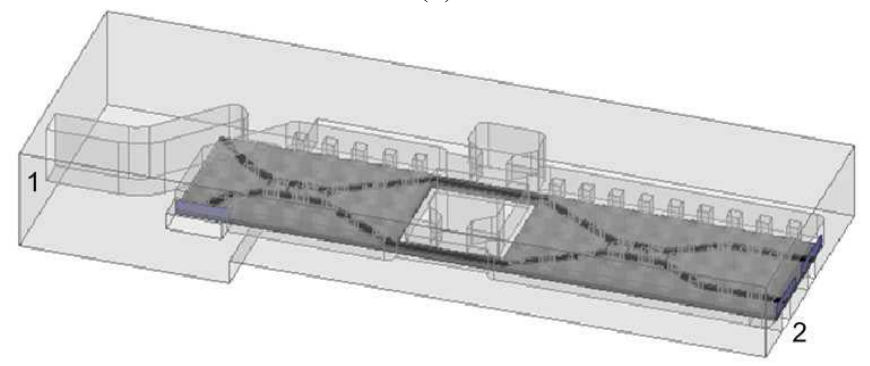

(b)

Fig. 12. Schematic diagram of the packaged W-band microstrip receiver. (a) Smooth top metal plate. (b) Pin surface on the upper metal plate.

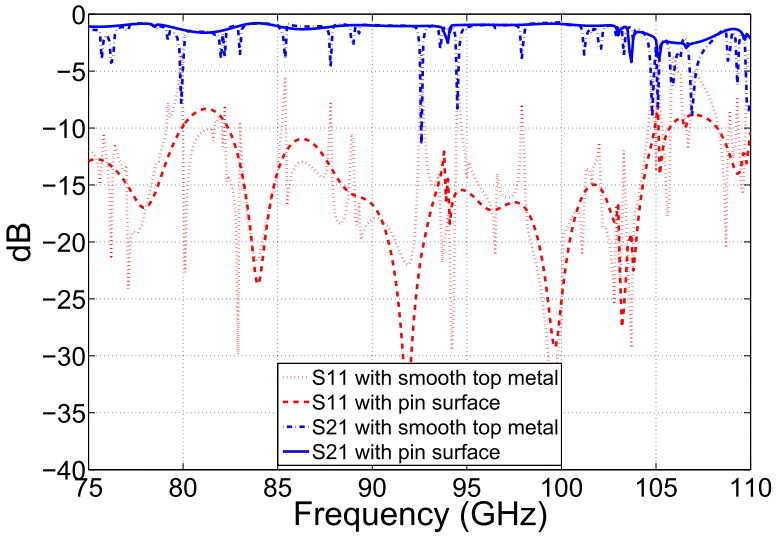

Fig. 13. Comparison between the case performance of the receiver when using a smooth surface metal package and a pin surface packaging.

in simulation using Ansoft HFSS. Two analysis have been carried out taken into account two packaging options; the first one uses a smooth surface in the upper metal plate, whereas in the second one, the pin surface is inserted (Fig. 12).

The comparison between transmission and reflection parameters of the W-band microstrip receiver inside this two packaging options is shown in Fig. 13. The simulation results demonstrate that the resonances caused by the undesired modes within the cavity are removed when the pin surface is introduced in the upper metal plate of the cavity. The small losses observed in the transmission parameter (S21) are caused by the dielectric substrate.

\section{EXPERIMENTAL RESULTS}

The experimental verification of the structure shown in Fig. 12 would be interesting to demonstrate the removal of the cavity resonances using the pin surface in the upper metal plate of the packaging. However, this receiver configuration 


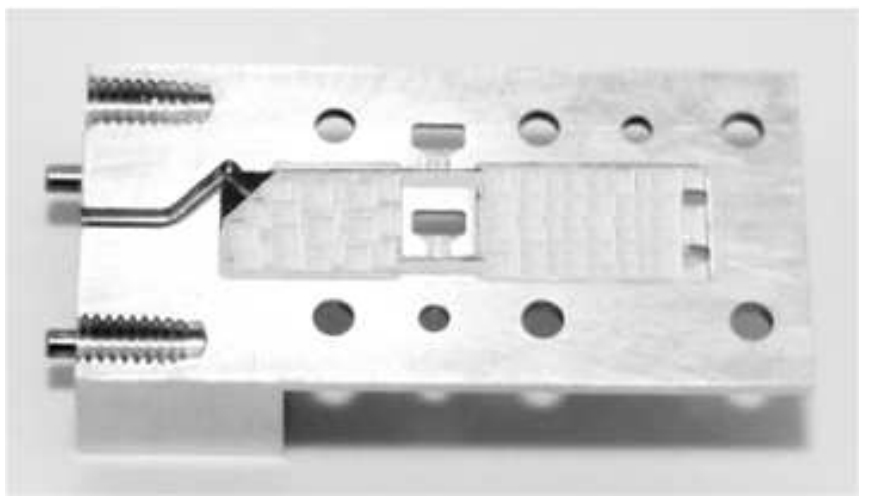

(a)

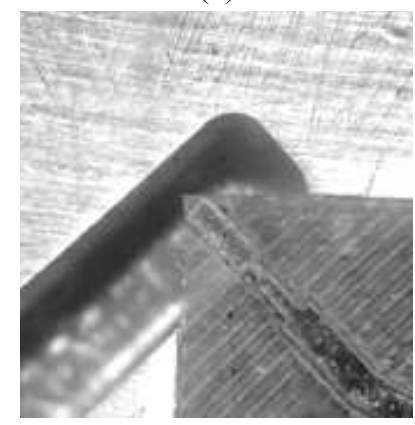

(b)

Fig. 14. Photographs of the bottom part of the manufactured packaged W-band microstrip receiver. (a) With a microstrip line. (b) Detail of the transition from WR-10 to microstrip.

has two output ports that are connected to MMIC detectors in order to convert the RF signal into dc and is not suitable for this experiment. For this reason, an alternative circuit has been used in order to verify the pin surface behavior. In order to excite the resonances, a short microstrip line has been used (Fig. 14). It consists of a transition from WR-10 waveguide to microstrip and a $50-\Omega$ microstrip line ended in open circuit. This open-circuited line excites the resonances produced by the cavity. Thereby, the removal of these resonances with the pin surface can be checked by measuring reflection at the waveguide input port.

The packaging metal block for the W-band microstrip receiver has been manufactured in aluminum using high precision milling. This package consists of two parts that are easily mounted through alignment pins and screws. The cutting plane of the packaging metal block in these two parts has been performed in accordance with the E-plane cut of the input waveguide to minimize losses. The bottom part has a cavity where the microstrip circuit is placed (Fig. 14). Two different top parts have been manufactured in order to demonstrate the removal of the undesired resonances. The first one contains the smooth surface, whereas the second one implements the pin surface on its upper metal plate. Their photographs are shown in Fig. 15(a) and (b). The short microstrip line was manually aligned and welded with silver epoxy to the bottom part, as shown in Fig. 14(b).

Fig. 16 shows the comparison between the simulation and the measurement results of this packaged W-band microstrip line for the two packaging options. Some manufacturing errors

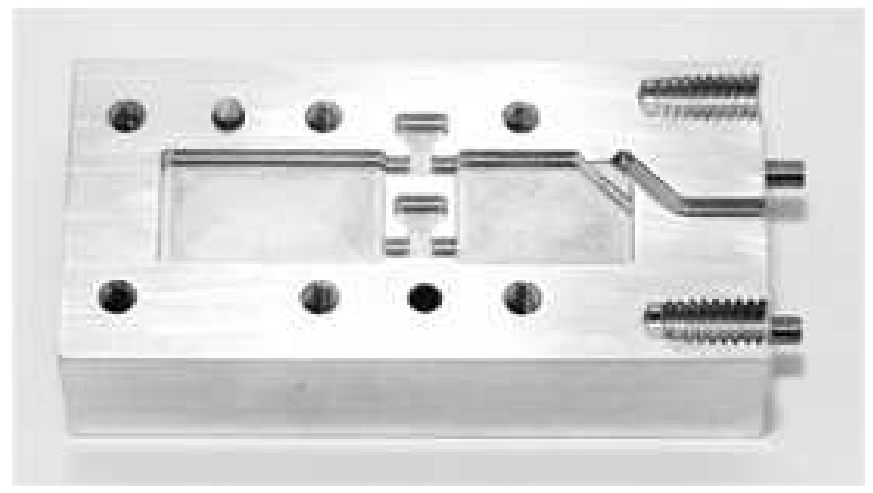

(a)

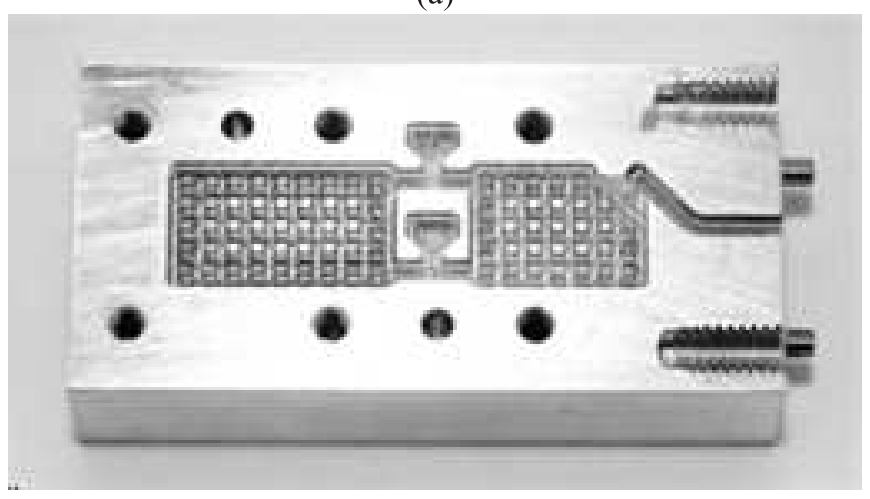

(b)

Fig. 15. Photographs of the top part of the manufactured packaged W-band microstrip receiver with (a) smooth metal surface and (b) pin surface.

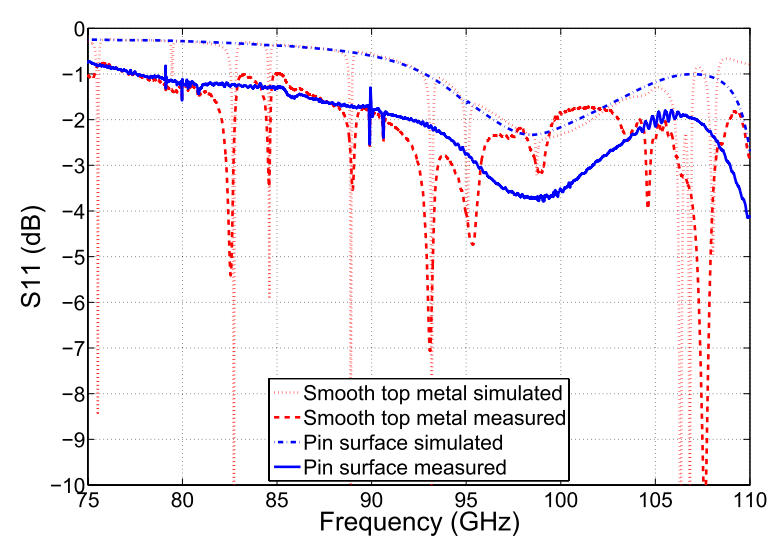

Fig. 16. Comparison between the simulated and measured results of the S11 parameter in the two packaging options: smooth metal and pin surface covers. Manufacturing errors in the microstrip circuit and in its position with respect to the input waveguide have been included.

in the microstrip circuit and also in its position have been detected and included in the simulation. In particular, the most relevant error is the $300-\mu \mathrm{m}$ displacement of the microstrip circuit in the transition, caused by the manual assembly. As can be seen, most of the resonances obtained with HFSS in the case of the smooth cavity appear in the measurements. In addition, the experiment confirms the removal of the resonances when the pin surface is used. Good agreement has been obtained in terms of the position of the resonances. However, there is a nearly constant difference in the reflection level of $1 \mathrm{~dB}$, which can be ascribed to losses in the metal 
waveguide section. Apart from this difference, these results demonstrate experimentally that the pin surface removes the unwanted resonances.

\section{CONCLUSION}

In this paper, the optimization of a pin surface has been carried out in order to suppress the propagation of undesired resonances in a packaged W-band microstrip circuit. This optimization has taken into account all the propagation directions. The performance of the optimized pin surface has been demonstrated by simulation in Ansoft HFSS. The experimental comparison between the use of a smooth cavity and a lid of pins to package a microstrip circuit also demonstrates the removal of the resonances along the entire W-band.

\section{REFERENCES}

[1] T. A. Midford, J. J. Wooldridge, and R. L. Sturdivant, "The evolution of packages for monolithic microwave and millimeter-wave circuits," IEEE Trans. Antennas Propag., vol. 43, no. 9, pp. 983-991, Sep. 1995.

[2] B. R. Hallford and C. E. Bach, "Lid interaction protected shield enclosed dielectric mounted microstrip," U.S. Patent 3638148 , Jan. 25, 1972.

[3] P.-S. Kildal and A. Kishk, "EM modeling of surfaces with STOP or GO characteristics-artificial magnetic conductors and soft and hard surfaces," Appl. Comput. Electromagn. Soc. J., vol. 18, no. 1, pp. 32-40, Mar. 2003.

[4] D. Sievenpiper, L. Zhang, R. F. Jimenez Broas, N. G. Alexopolous, and E. Yablonovitch, "High-impedance electromagnetic surfaces with a forbidden frequency band," IEEE Trans. Microw. Theory Technol., vol. 47, no. 11, pp. 2059-2074, Nov. 1999.

[5] E. Pucci, E. Rajo-Iglesias, and P.-S. Kildal, "New microstrip gap waveguide ion mushroom-type EBG for packaging of microwave components," IEEE Microw. Wireless Compon. Lett., vol. 22, no. 3, pp. 129-131, Mar. 2012.

[6] M. Silveirinha, C. Fernandes, and J. Costa, "Electromagnetic characterization of textured surfaces formed by metallic pins," IEEE Trans. Antennas Propag., vol. 56, no. 2, pp. 405-415, Feb. 2008.

[7] E. Rajo-Iglesias, P.-S. Kildal, A. U. Zaman, and A. Kishk, "Bed of springs for packaging of microstrip circuits in the microwave frequency range," IEEE Trans. Compon., Packag. Manuf. Technol., vol. 2, no. 10, pp. 1623-1628, Oct. 2012.

[8] D. Dawn, Y. Ohashi, and T. Shimura, "A novel electromagnetic bandgap metal plate for parallel plate mode suppression in shielded structures," IEEE Microw. Wireless Compon. Lett., vol. 12, no. 5, pp. 166-168, May 2008.

[9] P.-S. Kildal, E. Alfonso, A. Valero-Nogueira, and E. Rajo-Iglesias, "Local metamaterial-based waveguides in gaps between parallel metal plates," IEEE Antennas Wireless Propag. Lett., vol. 8, no. 1, pp. 84-87, Apr. 2009.

[10] P.-S. Kildal, A. U. Zaman, E. Rajo-Iglesias, E. Alfonso, and A. Valero-Nogueira, "Design and experimental verification of ridge gap waveguide in bed of nails for parallel-plate mode suppression," IET Microw. Antennas Propag., vol. 5, no. 3, pp. 262-270, Feb. 2011.

[11] E. Rajo-Iglesias and P.-S. Kildal, "Numerical studies of bandwidth of parallel-plate cut-off realised by a bed of nails, corrugations and mushroom-type electromagnetic bandgap for use in gap waveguides," IET Microw. Antennas Propag., vol. 5, no. 3, pp. 282-289, Feb. 2011.

[12] S. Rahiminejad et al., "Micromachined ridge gap waveguide and resonator for millimeter-wave applications," Sens. Actuators A, Phys., vol. 186, pp. 264-269, Oct. 2012.

[13] A. U. Zaman, J. Yang, and P.-S. Kildal, "Using lid of pins for packaging of microstrip board for descrambling the ports of eleven antenna for radio telescope applications," in Proc. IEEE APSURSI, Jul. 2010, pp. $1-4$.

[14] A. A. Brazalez, A. U. Zaman, and P.-S. Kildal, "Improved microstrip filters using PMC packaging by lid of nails," IEEE Trans. Compon. Packag. Manuf. Technol., vol. 2, no. 7, pp. 1075-1084, Jul. 2012.

[15] A. U. Zaman, M. S. Ellis, and P.-S. Kildal, "Metamaterial based packaging method for improved isolation of circuit elements in microwave module," in Proc. 7th EuMC, Oct. 2012, pp. 834-837.
[16] E. Rajo-Iglesias, A. U. Zaman, and P.-S. Kildal, "Parallel plate cavity mode suppresion in microstrip circuit packages using a lid of nails," IEEE Microw. Wireless Compon. Lett., vol. 20, no. 1, pp. 31-33, Jan. 2010.

[17] A. Rebollo, I. Maestrojuan, B. Larumbe-Gonzalo, R. Gonzalo, and I. Ederra, "Design and characterization of W-band components in planar technology," in Proc. 6th EuCAP, 2012, pp. 968-971.

[18] J. D. Joannopoulos, R. D. Meade, and J. N. Finn, Photonic Crystals: Molding the Flow of Light. Princeton, NJ, USA: Princeton Univ. Press, 1995.

[19] A. Rebollo, I. Maestrojuan, R. Gonzalo, and I. Ederra, "A broadband radiometer configuration at $94 \mathrm{GHz}$ in planar technology," in Proc. IMWS, 2011, pp. 89-92.

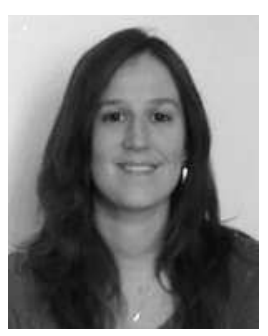

Ainara Rebollo was born in Pamplona, Spain, in 1983. She received the M.Sc. degree in telecommunication engineering from the Public University of Navarra (UPNA), Pamplona, Spain, in 2010, where she is currently pursuing the Ph.D. degree.

She is currently with the Antenna Group at UPNA. Her current research interests include passive imaging systems at millimeter-wave frequencies and their associated components in planar technology.

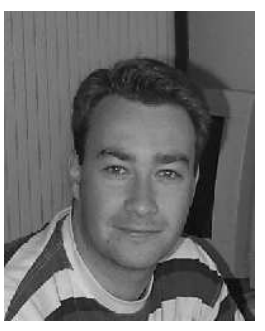

Ramón Gonzalo (S'95-M'00) was born in Logroño, La Rioja. He received the M.Sc. (Hons.) and Ph.D. (Hons.) degrees in ingeniero de telecomunicacion from the Public University of Navarra (UPNA), Pamplona, Spain.

$\mathrm{He}$ has been with the Antennas Group, Electrical and Electronic Engineering Department, UPNA, since 1995, where he is currently a Full Professor. From 1997 to 1998, he was a Research Fellow to the Antenna Section with the European Space Agency, European Space Research and Technology Centre, Noordwijk, The Netherlands, where he was involved in the modeling and design of electromagnetic crystal devices at microwave and millimeter-wave frequencies. From 2006 to 2008, he was a Sub-Director of the Engineering Faculty and, from 2008 to 2010, was the Head of Electrical and Electronic Engineering Department. He has been involved in more than 25 research projects at the European, and national level, acting as a co-ordinator in several of them. In particular, he has been a co-ordinator of five projects funded by the European Space Agency, three projects in the framework of the European Commission, and several of them funded by the Spanish Minister of Science. He has more than 70 journal publications in peerreview magazines and 120 conference papers related to his research lines. His current research interests include terahertz technologies, subsystems and devices, electromagnetic bandgap technology with emphasis on space antenna applications, design of waveguide transmission lines, and corrugated horn antennas.

Dr. Gonzalo was a co-recipient of the LAPC 2006, LAPC 2007, and IWAT 2007 Best Paper Awards.

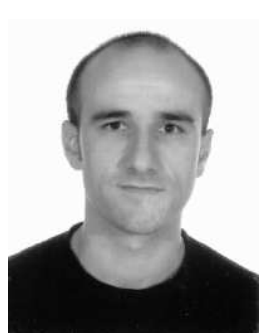

Iñigo Ederra was born in Isaba, Spain, in 1972. He received the Ingeniero de Telecomunicacion and $\mathrm{Ph} . \mathrm{D}$. degrees from the Universidad Pública de Navarra, Pamplona, Spain, in 1996 and 2004, respectively.

He joined the Microwave and Millimeter-Wave Group, Universidad Pública de Navarra, in 1997. From 1999 to 2000, he was with the European Space Agency, European Space Research and Technology Centre, Noordwijk, The Netherlands, where he was involved in electromagnetic bandgap materials and their applications in antennas. Since 2001, he has been with the Antenna Group, Universidad Pública de Navarra. In 2002, he was a Visitor Scientist with the Rutherford Appleton Laboratory, Didcot, U.K., participating in the Startiger project. His current research interests include electromagnetic bandgap materials and metamaterials, and their applications in microwave and millimeter-wave components and antennas.

Dr. Ederra was a co-recipient of the LAPC 2006 and IWAT 2007 Best Paper Awards. 